\title{
Lesiones nerviosas en extremidad pélvica y su manejo en un centro de referencia en la Ciudad de México
}

\author{
Lower extremity nerve injuries and their management at a referral center in Mexico City
}

\author{
José E. Telich-Tarriba ${ }^{1,2 *}$, Lilia C. de la Maza-Krzeptowsky³ y Alexander Cárdenas-Mejía1,2 \\ ${ }^{1}$ División de Cirugía Plástica y Reconstructiva, Hospital General Dr. Manuel Gea González; ${ }^{2}$ División de Estudios de Posgrado, Facultad de Medicina, \\ Universidad Nacional Autónoma de México; ${ }^{3}$ Departamento de Neurofisiología, Hospital Ángeles Pedregal. Ciudad de México, México
}

\begin{abstract}
Resumen
Objetivo: Describir el manejo de las lesiones nerviosas de extremidad inferior atendidas en un centro de referencia en cirugía plástica y reconstructiva en la Ciudad de México. Método: Estudio descriptivo, transversal y retrospectivo, en el que se evaluaron los expedientes clínicos de pacientes atendidos por lesiones nerviosas de extremidad inferior entre 2010 y 2018. Resultados: Se incluyeron 21 pacientes, 14 hombres (66.6\%) y 7 mujeres (33.3\%), con una edad promedio de $30.00 \pm 8.89$ años. Las lesiones en la extremidad derecha fueron más prevalentes (52.3\%). El mecanismo de lesión más común fue por herida por proyectil de arma de fuego (28.5\%) o por accidente automovilístico (13.6\%). Los nervios más afectados fueron el ciático (38.0\%) y el peroneo (38.0\%), seguidos por el tibial (9.5\%), el peroneo y el tibial simultáneamente (9.5\%) y el femoral (4.7\%). Las medidas de tratamiento más empleadas fueron las transferencias nerviosas (7 casos, 33.3\%) y la aplicación de injertos nerviosos para el manejo de brechas (7 casos, 33.3\%). Conclusiones: La reparación de las estructuras nerviosas de la extremidad inferior ha recibido poca atención. Sin embargo, nuestro estudio muestra que es posible aplicar diversas estrategias reconstructivas microquirúrgicas, buscando mejorar los desenlaces funcionales de los pacientes.
\end{abstract}

Palabras clave: Extremidad inferior. Lesiones de nervio periférico. Lesiones en pierna. Nervio femoral. Nervio peroneo. Transferencia nerviosa.

\section{Abstract}

Objective: To describe the management of lower extremity nerve injuries at a plastic and reconstructive surgery referral center in Mexico City. Method: A descriptive, transversal and retrospective study was performed, evaluating clinical records of patients with lower extremity nerve injuries managed in our center between 2010 and 2018. Results: Twenty-one patients were included, 14 males (66.6\%) and 7 females (33.3\%); mean age was $30.00 \pm 8.89$ years. Right-sided injuries were more prevalent (52.3\%). The most common etiologies were gunshot injuries (28.5\%) and motor vehicle accidents (13.6\%). The most frequently affected nerves were the sciatic (38.0\%) and the peroneal nerves (38.0\%), followed by the tibial (9.5\%), simultaneous tibial and peroneal nerve injury (9.5\%) and the femoral nerve (4.7\%). Nerve transfers (7 cases, 33.3\%) and autologous nerve grafts (7 cases, 33.3\%) were the preferred surgical techniques for injury management. Conclusions: Repair of lower

\footnotetext{
Correspondencia:

*José E. Telich-Tarriba

Avda. Calzada de Tlalpan, 4800

Col. Belisario Domínguez, Sec. 16, Del. Tlalpan Fecha de recepción: 22-03-2020

C.P. 14080, Ciudad de México, México Fecha de aceptación: 28-09-2020

Cir Cir. 2020;89(2):200-205

E-mail: josetelich@gmail.com

DOI: $10.24875 / C I R U .20000211$

Contents available at PubMed

www.cirugiaycirujanos.com

0009-7411/@ 2020 Academia Mexicana de Cirugía. Publicado por Permanyer. Este es un artículo open access bajo la licencia CC BY-NC-ND (http://creativecommons.org/licenses/by-nc-nd/4.0/).
} 
extremity nerve injuries has not been popularized, however our study shows that several microsurgical reconstructive strategies are available in order to improve functional outcomes.

Key words: Lower extremity. Peripheral nerve injuries. Leg injuries. Femoral nerve. Peroneal nerve. Nerve transfer.

\section{Introducción}

El trauma de extremidades inferiores es muy común y potencialmente incapacitante. En los Estados Unidos de Norteamérica, el National Trauma Data Bank reportó 278,100 casos en 2012, lo que representa el $14 \%$ del total de las consultas en los servicios de urgencias $^{1,2}$. De forma similar, el Instituto Nacional de Salud Púbica de México reporta que los traumatismos en las extremidades inferiores representaron el 13\% de las consultas por lesiones en los sistemas hospitalarios públicos, superadas únicamente por los traumatismos en las extremidades superiores ${ }^{3}$.

El tratamiento de estos pacientes requiere un manejo multidisciplinario coordinado que incluye la colaboración de cirujanos plásticos. Actualmente existen protocolos de tratamiento bien establecidos para la reconstrucción de la cubierta cutánea y las estructuras óseas de la extremidad inferior, pero este no es el caso en el manejo de las lesiones asociadas con daño a nervios periféricos ${ }^{4}$.

Las lesiones nerviosas en la extremidad inferior ocasionan alteraciones en la sensibilidad y la movilidad, limitando la capacidad de las personas para deambular, ejercitarse o trabajar, lo que afecta profundamente su calidad de vida y las interacciones sociales o laborales. En Europa se ha reportado que el 2\% de los pacientes que sufren un trauma de extremidad inferior pueden presentar una lesión nerviosa concomitante ${ }^{5}$. Es posible que la incidencia sea mayor debido a que, en este grupo de pacientes, la prioridad de atención suele enfocarse en el manejo del politrauma, por lo que las lesiones pasan desapercibidas 0 se diagnostican de forma tardía. En nuestro país se desconoce la incidencia de este tipo de lesiones; sin embargo, un informe del Hospital Universitario Dr. José Eleuterio González, en Monterrey, reportó que las lesiones de nervio periférico constituyeron el 1.12\% del total de las consultas atendidas en su servicio de urgencias, de las cuales el $15 \%$ se presentaron en las extremidades inferiores $(0.16 \% \text { del total })^{6}$.

En el entorno urbano, las principales causas de lesiones en las extremidades inferiores son los traumatismos cerrados asociados a accidentes en vehículos de motor, seguidos por caídas, laceraciones y heridas por proyectil de arma de fuego. Estas lesiones afectan principalmente a hombres $(80 \%)$ y en edades productivas (16 a 59 años, $86 \%)^{5}$.

La evaluación clínica debe enfocarse inicialmente en los principios de estabilización del paciente de trauma, y luego, una vez que el paciente se encuentre alerta y sea capaz de cooperar, deberá llevarse a cabo una exploración clínica neurológica exhaustiva para identificar alteraciones motoras y sensitivas en la extremidad, centrándose en especial en los nervios femoral, ciático, peroneo y tibial:

- Nervio femoral: las lesiones del nervio femoral causan hipoestesia en la cara anterior del muslo y debilidad para la flexión de la cadera y la extensión de la rodilla.

- Nervio ciático: el daño al nervio ciático reduce la sensibilidad en la porción lateral de la pierna, así como en el dorso, la planta y la región lateral del pie; se acompaña también de debilidad en la flexión de la rodilla y de incapacidad para mover el pie.

- Nervio peroneo: su lesión reduce la sensibilidad en el primer espacio interdigital e impide la flexión dorsal del pie.

- Nervio tibial: su lesión ocasiona pérdida de sensibilidad en el talón e incapacidad para realizar flexión plantar del pie?.

El interés por realizar reconstrucciones nerviosas de extremidad inferior no se ha extendido, en primer lugar porque tradicionalmente no se considera que la sensibilidad y la movilidad de esta sean tan críticas y necesarias como en la extremidad superior, y en segundo lugar debido a que la mayor longitud de la extremidad retarda la reinervación de los órganos diana, lo que puede ocasionar resultados poco favorables. El manejo tradicional de estas lesiones ha recaído principalmente en el área de la ortopedia, enfocándose en el uso de ortesis o en la realización de procedimientos estáticos, como artrodesis de tobillo y transferencias tendinosas o musculares ${ }^{8}$.

Los cirujanos de nervio periférico actualmente cuentan con un vasto arsenal terapéutico para el manejo de las lesiones nerviosas, los cuales van desde neurorrafias simples hasta la construcción de transferencias nerviosas complejas: 
- Neurorrafia: la aproximación de los muñones del nervio lesionado y su coaptación con suturas de nailon de 8 a 10-0 es el método de referencia para la reconstrucción nerviosa. Actualmente se emplean dos técnicas, las suturas epineurales y la reparación por grupos fasciculares, y ambas han demostrado ser igualmente efectivas siempre y cuando los muñones contengan fascículos viables y la reparación se lleve a cabo sin tensión $n^{9,10}$.

- Injerto nervioso: está indicado cuando no es posible realizar una reparación primaria sin tensión, en especial en brechas mayores de $3 \mathrm{~cm}$. Los nervios donadores deben ser fácilmente accesibles y causar poca morbilidad; los más utilizados son los nervios sural $(30-40 \mathrm{~cm})$, safeno interno $(40 \mathrm{~cm})$, cutáneo femoral lateral $(30 \mathrm{~cm})$ y cutáneos antebraquiales mediales $(20 \mathrm{~cm})$. Los resultados posoperatorios son poco satisfactorios en caso de brechas nerviosas mayores de $6 \mathrm{~cm}$ y en lesiones muy proximales ${ }^{11}$.

- Injerto nervioso vascularizado: ampliamente descrito por la Dra. Julia Terzis ${ }^{12}$. Está indicado cuando se cuenta con un lecho receptor mal vascularizado o se requiere un injerto nervioso demasiado largo $(>6 \mathrm{~cm})$. El más utilizado es el nervio cubital, aunque también se han empleado otros, como el sural y el safeno. Cuenta con la ventaja de acelerar el ritmo de crecimiento axonal a $3 \mathrm{~mm}$ por día ${ }^{13}$.

- Transferencia nerviosa: sacrifica nervios con función motora o sensitiva redundante para convertir una lesión proximal en el nervio receptor en una lesión distal del nervio donador. Sus principales indicaciones son lesiones muy proximales, reparaciones tardías, avulsión de raíces nerviosas o presencia de brechas nerviosas muy amplias. El nervio donador ideal debe ser cercano al órgano diana, tener una función sinérgica con la musculatura que se va a reinervar y contar con una carga axonal similar a la del nervio receptor ${ }^{14}$.

En forma análoga a la reconstrucción del plexo braquial, existen prioridades funcionales al realizar una reconstrucción nerviosa en la extremidad inferior. La estabilidad y la extensión de la rodilla son la primera meta (reinervación del vasto medial), seguidas por la flexión plantar del pie, lograda por el nervio tibial, al igual que la recuperación de la sensibilidad plantar. Finalmente, la recuperación de la dorsiflexión del pie por parte del nervio peroneo profundo permitirá mejorar la deambulación, evitando la caída del pie ${ }^{15}$.
Es importante señalar que la literatura internacional sobre este tema está compuesta principalmente por reportes de casos, series de casos pequeñas, disecciones cadavéricas y revisiones del tema basadas en la experiencia de autores de renombre ${ }^{11}$.

El Hospital General Dr. Manuel Gea González es un centro de referencia nacional e internacional para cirugía plástica, especialmente dentro de los campos de la cirugía craneofacial, microcirugía y reconstrucción de nervio periférico ${ }^{16}$. El objetivo del presente trabajo es describir las lesiones nerviosas de extremidad inferior atendidas en nuestro servicio.

\section{Método}

Estudio observacional, descriptivo, transversal y retrospectivo en el que se evaluaron expedientes clínicos de pacientes atendidos por lesiones nerviosas de extremidad inferior en la división de cirugía plástica y reconstructiva del Hospital General Dr. Manuel Gea González, en el periodo entre enero de 2010 y diciembre de 2018.

La información fue recolectada en una base de datos en Microsoft Excel incluyendo la edad, el sexo, el nervio lesionado, el mecanismo de la lesión y el tipo de tratamiento otorgado.

Se utilizó estadística descriptiva para evaluar las características demográficas y clínicas de los pacientes. Las variables continuas se presentan como medidas de tendencia central, y las categóricas como totales y porcentajes.

\section{Resultados}

Durante el periodo de estudio se atendieron 21 pacientes con lesiones nerviosas de extremidad inferior, lo que representa el $0.002 \%$ del total de los pacientes operados y el $13 \%$ de las lesiones de extremidad inferior atendidas en nuestro servicio en dicho periodo. Catorce (66.6\%) pacientes fueron hombres y 7 (33.3\%) fueron mujeres, resultando en una relación hombre:mujer de 2:1.

La edad promedio de presentación fue de $30.00 \pm$ 8.89. años y la distribución de edades fue la siguiente: $3(14.2 \%)$ pacientes eran menores de 15 años, 10 (47.6\%) tenían entre 16 y 30 años, 4 (19.0\%) tenían entre 31 y 45 años, y 4 (19.0\%) tenían entre 46 y 60 años.

Las lesiones en la extremidad derecha fueron ligeramente más prevalentes que en la extremidad derecha $(52,3$ vs. $47.6 \%)$. El mecanismo de lesión más 
común fueron las heridas por proyectil de arma de fuego, con $6(28.5 \%)$ casos, seguidas de los accidentes automovilísticos en $3(13.6 \%)$, la resección de tumores nerviosos en 3 (13.6), y iatrogénicas en 2 $(9.5 \%)$; el resto $(33.3 \%)$ de los mecanismos del trauma fueron desconocidos.

Los nervios afectados, en orden decreciente, fueron el ciático en $8(38.0 \%)$ casos, el peroneo en 8 (38.0\%), el tibial en $2(9.5 \%)$, el peroneo y el tibial simultáneamente en 2 (9.5\%), y el femoral en 1 (4.7\%) (Tabla 1).

Todos los pacientes fueron operados empleando monitoreo neurofisiológico transoperatorio ${ }^{17}$. Las medidas de tratamiento más empleadas fueron las transferencias nerviosas (7 casos, 33.3\%) y la aplicación de injertos nerviosos para el manejo de brechas (7 casos, 33.3\%). En $4(19.0 \%)$ pacientes se realizó exploración nerviosa y neurólisis, y 2 (9.5\%) fueron manejados únicamente con rehabilitación. Dos (9.5\%) pacientes optaron por no recibir tratamiento en la institución.

Al seguimiento se observó que el $71.4 \%$ de los pacientes con transferencias nerviosas lograron una recuperación M4 y el 28.5\% alcanzaron M3, mientras que en el grupo de autoinjertos el $42.8 \%$ alcanzaron M3 y solo el $28.5 \%$ llegaron a M4.

\section{Discusión}

Las lesiones de nervio periférico son un problema frecuente que condiciona una disfunción de las extremidades, resultando en un impacto negativo en la calidad de vida de los pacientes, así como una importante carga social y económica. La reparación de las lesiones nerviosas es un proceso fisiopatológico complejo en el que se involucran la degeneración Walleriana, la regeneración axonal y factores neurotróficos y neurotrópicos, todo con el fin de evitar la atrofia de la placa neuromotora y muscular. La meta de toda reconstrucción nerviosa es conseguir la reinervación de los órganos diana mediante una técnica quirúrgica cuidadosa, libre de tensión y con la menor pérdida de fibras nerviosas en las líneas de sutura ${ }^{18}$.

A pesar del auge que la cirugía de nervio periférico ha experimentado en las últimas décadas en los campos de reconstrucción del plexo braquial y del nervio facial, logrando resultados confiables y predecibles $^{15}$, la reconstrucción nerviosa de las extremidades inferiores ha sido poco estudiada, por lo que los pacientes con frecuencia son manejados con ortesis, transferencias tendinosas, artrodesis e incluso amputaciones ${ }^{19,20}$.
Los primeros casos de reconstrucción nerviosa en las extremidades inferiores fueron reportados durante la Segunda Guerra Mundial, con resultados poco alentadores $^{21}$. Fue a finales de la década de 1980 cuando surgió de nuevo el interés por la reparación de lesiones nerviosas de miembros inferiores, ampliando las aplicaciones de los autoinjertos y posteriormente desarrollando técnicas novedosas, como el uso de injertos nerviosos vascularizados y de transferencias nerviosas ${ }^{11}$.

La principal limitante para la recuperación en este tipo de lesiones es la distancia que las fibras nerviosas deben recorrer para alcanzar los músculos diana, resultando en tiempos de reinervación prolongados, en especial si se trata de lesiones de localización proximal14. Otras causas importantes son el tiempo de evolución y la presencia de brechas nerviosas; los pacientes con más de 1 año de evolución o con brechas mayores de $6 \mathrm{~cm}$ suelen tener peores resultados ${ }^{11}$.

Los estudios epidemiológicos sobre la prevalencia de las lesiones nerviosas en las extremidades inferiores reportan que varía entre el $0.8 \%$ y el $1.8 \%$, que afectan de manera predominante a los hombres (80 a $83 \%$ ) y con mayor frecuencia al nervio peroneo (aproximadamente 50\%). Los mecanismos del trauma varían dependiendo del origen de los reportes, pero los más comunes son los accidentes automovilísticos y las heridas cortantes ${ }^{5,22}$. En nuestro país, la epidemiología de este tipo de lesiones de nervio periférico es limitada. El único reporte existente es el publicado por Castillo-Galván, et al. ${ }^{6}$, quienes comunican 21 lesiones, siendo la mayoría secundarias a heridas punzocortantes y con mayor frecuencia en el nervio plantar.

En comparación con las series anteriores, en nuestros pacientes encontramos una mayor proporción de mujeres y de lesiones derivadas de heridas por proyectil de arma de fuego, y que los nervios más lesionados son el ciático y el peroneo. Estos resultados pueden diferir debido a que nuestra institución es un centro de referencia para cirugía reconstructiva, por lo que nuestra estadística puede no coincidir con la de centros especializados en trauma.

Hasta el momento no existe una técnica única de elección para la reparación nerviosa. Se han comparado los resultados entre distintas técnicas, pero los reportes existentes suelen limitarse a series relativamente pequeñas. Cada caso deberá individualizarse con el fin de realizar una reconstrucción anatómica, considerando factores como el nervio afectado, el tiempo de evolución y la presencia de brechas. Todo 
Tabla 1. Características demográficas de la población

\begin{tabular}{lcc}
\hline & $\mathbf{n}$ & $\%$ \\
\hline Sexo & & \\
Masculino & 14 & 66.6 \\
Femenino & 7 & 33.3 \\
Grupos de edad & & \\
$\quad$ 15 años & 3 & 14.2 \\
16-30 años & 10 & 47.6 \\
3-45 años & 4 & 19.0 \\
45-60 años & 4 & 19.0 \\
& & \\
Lado afectado & 11 & 52.3 \\
Derecho & 10 & 47.6 \\
Izquierdo & & \\
Nervio afectado & & 4.7 \\
Femoral & 1 & 38.0 \\
Ciático & 8 & 9.5 \\
Tibial & 2 & 38.0 \\
Peroneo & 8 & 9.5 \\
Tibial y peroneo & 2 & \\
\hline
\end{tabular}

paciente deberá ser evaluado en forma integral con una exploración clínica sensitiva y motora, tomando en cuenta los hallazgos de los estudios electrofisiológicos y de imagen ${ }^{20}$.

La evaluación neurofisiológica transoperatoria es actualmente parte integral de la evaluación y la reconstrucción de nervios periféricos. Su objetivo principal es localizar y caracterizar los sitios de lesión, verificar la viabilidad de los muñones nerviosos e identificar nervios donadores para la reconstrucción, permitiendo al cirujano familiarizado con la técnica objetivar la toma de decisiones reconstructivas ${ }^{23}$.

Las modalidades de tratamiento más empleadas en nuestro centro fueron la aplicación de injertos no vascularizados y las transferencias nerviosas. En nuestra serie observamos un cambio en las estrategias de tratamiento: antes de 2014, los pacientes solían ser manejados con puentes nerviosos, pero en la actualidad se prefiere el uso de transferencias debido a la proximidad anatómica de los nervios donadores y a la posibilidad de obtener mejores resultados en lesiones proximales o con tiempos de evolución largos. No se realizaron neurorrafias directas debido a que con frecuencia los pacientes son referidos en forma tardía.

Las principales limitantes de este trabajo son su naturaleza retrospectiva y la muestra pequeña. Sin embargo, se trata del primer reporte enfocado en el manejo de lesiones nerviosas de extremidad inferior en nuestro país, y sienta un precedente para el desarrollo de guías de manejo de este tipo de pacientes. Nuestras líneas de investigación futuras se centrarán en estudiar los desenlaces clínicos de los pacientes a largo plazo y comparar los resultados de cada modalidad de tratamiento, en especial del uso de injertos contra transferencias.

\section{Conclusiones}

La reparación de las estructuras nerviosas de la extremidad inferior ha recibido poca atención. Nuestro estudio ha mostrado que es posible aplicar diversas estrategias reconstructivas microquirúrgicas, buscando mejorar los desenlaces funcionales de los pacientes.

\section{Responsabilidades éticas}

Protección de personas y animales. Los autores declaran que para esta investigación no se han realizado experimentos en seres humanos ni en animales.

Confidencialidad de los datos. Los autores declaran que han seguido los protocolos de su centro de trabajo sobre la publicación de datos de pacientes.

Derecho a la privacidad y consentimiento informado. Los autores declaran que en este artículo no aparecen datos de pacientes.

\section{Conflicto de intereses}

Los autores declaran que no existen conflictos de intereses.

\section{Bibliografía}

1. Nance ML. National Trauma Data Bank Annual Report. 2012. (Consultado el 22 de octubre de 2013.) Disponible en: http://www.facs.org/ trauma/ntdb/pdf/ntdb-annual-report-2012.pdf.

2. Lambers K, Ootes D, Ring D. Incidence of patients with lower extremity injuries presenting to US emergency departments by anatomic region, disease category, and age. Clin Orthop Relat Res. 2012;470:284-90.

3. Ávila-Burgos L, Ventura-Alfaro C, Barroso-Quiab A, Aracena-Genao B, Cahuana-Hurtado L, Serván-Mori E, et al. Las lesiones por causa externa en México. Lecciones aprendidas y desafíos para el Sistema Nacional de Salud Mexicano. México 2010. Ciudad de México/Cuernavaca, México: Instituto Nacional de Salud Pública; 2010.

4. Ong YS, Levin LS. Lower limb salvage in trauma. Plast Reconstr Surg. 2009;125:582-7.

5. Huckhagel T, Nüchtern J, Regelsberger J, Gelderblom M, Lefering R; TraumaRegister DGU®. Nerve trauma of the lower extremity: evaluation of 60,422 leg injured patients from the TraumaRegister DGU® between 2002 and 2015. Scand J Trauma Resusc Emerg Med. 2018;26:40.

6. Castillo-Galván ML, Martínez-Ruiz FM, de la Garza-Castro O, Elizondo-Omaña RE, Guzmán-López S. [Study of peripheral nerve injury in trauma patients]. Gac Med Mex. 2014;150:527-32.

7. Cannon JW, Rasmussen TE. Severe extremity injury in the adult patient UpToDate; 2019. (Consultado el 14 de abril de 2019.) Disponible en: https://www.uptodate.com/contents/severe-extremity-injury-in -the-adult-patient\#H151204860.

8. O'Malley MP, Pareek A, Reardon P, Krych A, Stuart MJ, Levy BA. Treatment of peroneal nerve injuries in the multiligament injured/dislocated knee. J Knee Surg. 2016;29:287-92. 
9. Ghavami A, Benhaim P, Kallina CF. Nerve injuries. En: Janis J, editor. Essentials of plastic surgery. 2nd ed. Saint Louis, MO: Quality Medical Publishing Inc; 2014. p. 951-63.

10. Gürbüz Y, Sügün TS, Özaksar K, Kayalar M, Toros T, Ademoğlu Y. Peroneal nerve injury surgical treatment results. Acta Orthop Traumato Turc. 2012;46:438-42

11. Ray WZ, Mackinnon SE. Management of nerve gaps: autografts, allografts, nerve transfers, and end-to-side neurorrhaphy. Exp Neurol. 2010;223:77-85.

12. Breidenbach W, Terzis JK. The anatomy of free vascularized nerve grafts. Clin Plast Surg. 1984;11(1):65-71

13. Habre SB, Bond G, Jing XL, Kostopoulos E, Wallace RD, Konofaos P. The surgical management of nerve gaps: present and future. Ann Plast Surg. 2018;80:252-61.

14. Farnebo S, Thorfinn J, Dahlin LB. Peripheral nerve injuries of the upper extremity. En: Neligan PC, editor. Plastic surgery. 4th ed. Philadelphia: Elsevier; 2018. p. 714-39.

15. Aldea PA, Shaw WW. Management of acute lower extremity nerve injuries. Foot \& Ankle. 1986;7:82-94.

16. Telich-Tarriba JE, Velázquez E, Theurel-Cuevas A, Shinji-Pérez K, Anaya-Ayala JE, Jiménez-Murat $Y$, et al. Upper extremity patterns of injury and management at a plastic and reconstructive surgery referral center in Mexico City. Ann Plast Surg. 2018;80:23-6.
17. Maza-Krzeptowsky LC, Romero-Esquiliano G, Ramírez-Segura EH, Obieta-Cruz E, Vega-Sosa A, Cárdenas-Mejía A, e. [Implementation of intraoperative neurophysiologic monitoring in children and adults in secondary and tertiary health care facilities]. Cir Cir. 2018;86:132-9.

18. Jiang BG, Han N, Rao F, Wang YL, Kou YH, Zhang PX. Advance of peripheral nerve injury repair and reconstruction. Chin Med $J(E n g l)$. 2017;130:2996-8.

19. Nath RK, Lyons AB, Paizi M. Successful management of foot drop by nerve transfers to the deep peroneal nerve. J Reconstr Microsurg. 2008;24:419-27.

20. Iñigo-Arroyo F, Cárdenas-Mejía A. Reconstrucción nerviosa de extremidad inferior bajo monitoreo transoperatorio utilizando transferencias nerviosas e injertos nerviosos. Reporte de 3 casos. [Tesis]. México: Universidad Nacional Autónoma de México; 2018.

21. Kline DG. Operative management of major nerve lesions of the lower extremity. Surg Clin North Am. 1972;52:1247-65.

22. Murovic JA. Lower-extremity peripheral nerve injuries: a Louisiana State University Health Sciences Center literature review with comparison of the operative outcomes of 806 Louisiana State University Health Sciences Center sciatic, common peroneal, and tibial nerve lesions. Neurosurgery. 2009;65(4 Suppl):A18-A23.

23. García-García F, Cárdenas-Mejía A. Utilidad de la monitorización electrofisiológica transoperatoria en el abordaje de las lesiones de plexo braquial. Cir Plast Iberolatinoam. 2017;43:305-12. 